\title{
Solving for the Best Value of Bias Resistor to Promote Stability of Rs485 Fieldbus
}

\author{
Liang Zhao ${ }^{1 *}$, Ruobing Liang ${ }^{2}$ and Jili Zhang ${ }^{2}$ \\ ${ }^{1}$ Faculty of Electronic Information and Electrical Engineering, Dalian \\ University of Technology, Dalian 116024, China \\ ${ }^{2}$ Faculty of Infrastructure Engineering, Dalian University of Technology, \\ Dalian 116024, China \\ *Email: zliangdut@qq.com
}

\begin{abstract}
This paper analyzes several factors affecting the reliability of RS485 fieldbus communication, and then establishes the equivalent circuit model in order to solve bias

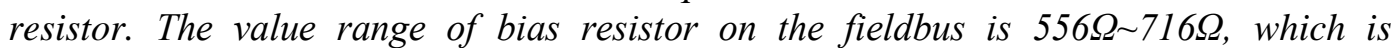
determined by using the law of KCL and current limiting method. Furthermore the best

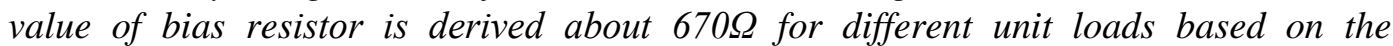
relationship between number of network nodes and the value of bias resistor, and the corresponding maximum network nodes of fieldbus are determined too. The research of this paper provides theoretical basis and engineering design guidance for the application of RS485 fieldbus.
\end{abstract}

Keywords: RS485 fieldbus, Bias resistor, Optimization method

\section{Introduction}

RS485 fieldbus is one of the most popular industry standard communication interface, which has been widely used in industrial field, traffic, lighting and power system, environmental monitoring, energy consumption monitoring, data acquisition system etc. [1-6], by its special advantage of low cost, multiple nodes, high ability of anti-interference and long transmission distance. However, most of the RS485 working scenes are with strong electromagnetic interference, which always leads to many communication problems. Therefore, how to improve the reliability of RS485 communication has been a hot spot in recent years.

Various researchers have exploited relevant methods to promote stability of RS485 fieldbus [7-10]. In 2004, Zhang et al. [7] reported their work from aspects of the RS485 chip selection, connection terms, photoelectric isolation, signal reflection and adding bias resistors, to enhance the communication reliability of the fieldbus. The research pointed out that, it did not need terminal resistors if the communication cable of RS485 fieldbus was not long. However, the judgment method of this distance was not mentioned in it. In 2006, Zhang et al. [8] provided several ways from the network topology, lead wire length, isolation and power supply to design the reliability of RS485 fieldbus. Similar to [7], Yu et al. [9] did a qualitative analysis for fail-safe and reflection interference to improve the stability of the RS485 bus. To our knowledge, the exiting literature to analyze values of key parameters of RS485 fieldbus in quantitative mode is in reference [10] in 2013 by Zhao et al. They calculated the theoretical value of bias resistor was about $570 \Omega$ based on KCL current equation. Their analyses showed that the maximum bus loading of RS485 fieldbus would be reduced after adding bias resistor and terminal resistor, however, the relevant quantitative results were not given. 
Our previous work [11], proposed the equivalent circuit model of RS485 fieldbus and calculated the theoretical minimum value of bias resistor, in addition, the influence of bias resistior on the maximum load and terinal resistor were discussed. On the basis of our previous research, the current context will do a further analysis on the structure design optimization and solve the value range of bias resistor, more important, the best value of bias resistor will be got. At the exact point, it can play the role of the bias resistor, namely, safeguarding the RS485 fieldbus communication stability, and can get the maximum bus nodes at the same time.

\section{Communication Reliability Influencing Factors Analysis}

There are some factors that influence the communication reliability of RS485 fieldbus, which can be divided into the following categories:

(1) Communication cable is too long:

The transmission cable length of RS485 bus should meet the requirements of standard. If the communication distance is too long, it will lead to the decrease of the quality of transmitted signal, and then cause bit error rate rising.

(2) The number of network nodes is too many:

The practical engineering application shows that, the maximum bus load is a constant value for a given chip of RS485 bus. If the network takes on more than the maximum number of nodes, it will decline the driving ability, and result in communication failure.

(3) Signal reflection interference:

By the transmission line theory, when the signal during transmission arrives at the end of the twisted pair, if encounters impedance incontinuity or impedance mutation, it will cause signal reflection [12]. The signal reflection will lead to signal waveform distortion, decreasing of the signal quality and even communication failure of RS485 fieldbus.

(4) The influence of "False Start Signal (FSS)"

Fig. 1 shows the data transmission process of RS485 fieldbus, which adopts 1 bit low level signal as the start of data transmission, and 1 bit high level signal as a termination signal. In this context, when the RS485 bus is idle, if bursting in a low level signal, slave nodes on the bus will regard it as a start flag, and try to receive data. However, because the bus is in idle state, and there is no real data to send, so there will not be a stop bit. The FSS phenomenon will occupy the bus, and make the communication disabled.

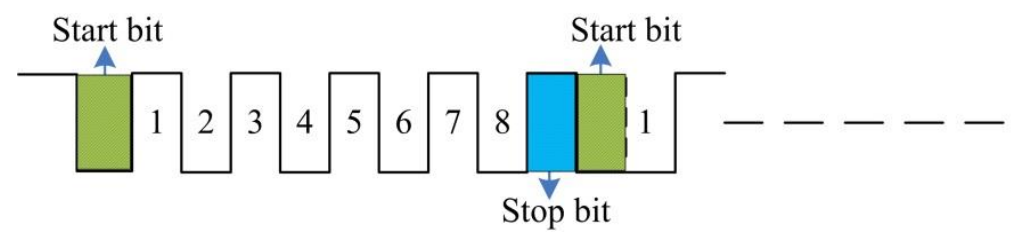

Figure 1. Data Transmission Format of RS485 Bus

In all of influencing factors as analyzed above, the cable length and number of network nodes can be controlled when cabling. As for the signal reflection, it could be solved by adding two terminal resistors $R_{\mathrm{T}}$ on the each end of the RS485 fieldbus, which will keep the impedance continuity. However, as for the phenomenon of FSS, it could be decreased by adding two bias resistors $R_{\mathrm{B}}$, which could keep the bus at a determining high level state when the bus is idle. Fig. 2 shows the final network structure after optimization, with two bias resistors on the A and B transmission line, $R_{\mathrm{T} 1}$ and $R_{\mathrm{T} 2}$ on the two end of RS485 bus, respectively. 


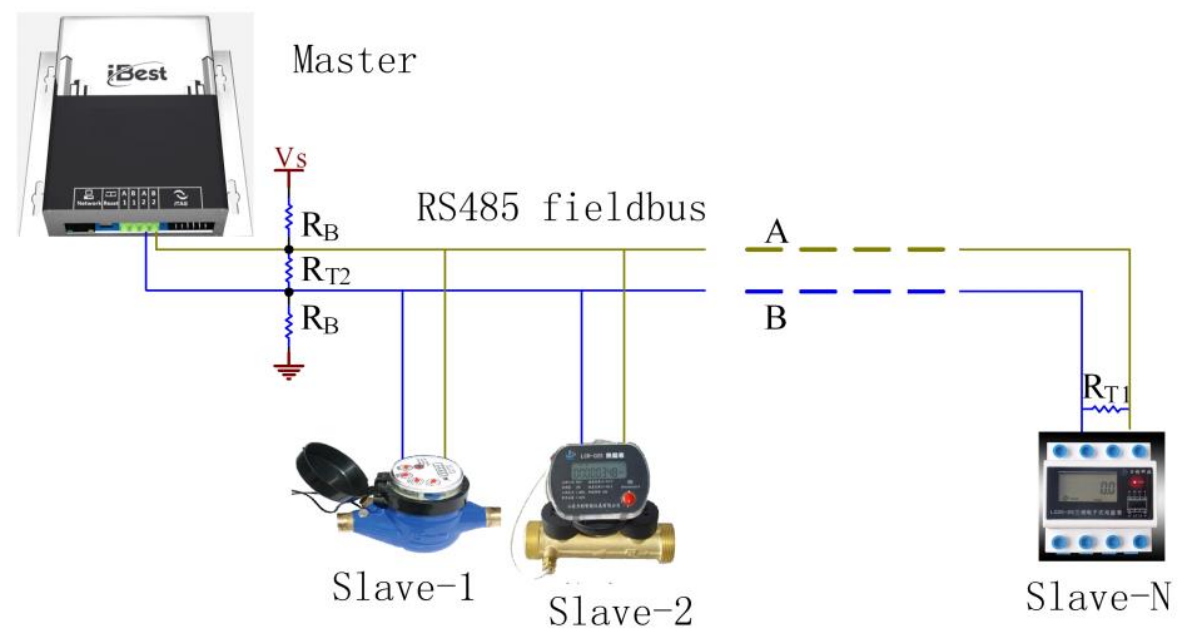

Figure 2. RS485 Network with Biasing Resistors and Terminal Resistor

\section{Equivalent Circuit Model}

\subsection{The Equivalent Circuit of RS485 Fieldbus}

It is essential to transform the physical model into equivalent circuit model, in order to analyze the parameters of RS485 fieldbus. As shown in Fig.3, the master and salves that mounted on the bus could be equivalent as input resistances on the transmission lines. In this context, the values of terminal resistance $R_{\mathrm{T}}$ and bias resistors $R_{\mathrm{B}}$ could be solved.

For convenience of theoretical derivation, and combined with the practical engineering application experience, some assumptions are as following:

(1) The network topology of RS485 fieldbus is standard, namely, "hand in hand".

(2) The highest communication rate is not higher than $9600 \mathrm{bps}$.

(3) The cable length is less than $1200 \mathrm{~m}$;

(4) The number of network nodes is within the maximum nodes.

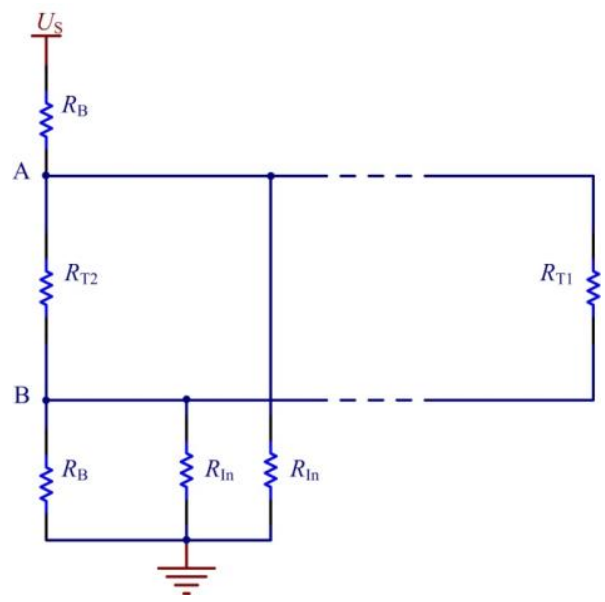

Figure 3. Equivalent Circuit of RS485 Network with Failsafe Biasing Resistors 


\subsection{The Constraint Conditions}

(1) The minimum threshold voltage

RS485 fieldbus defines the threshold voltage of the receiver is $200 \mathrm{mV}$, based on this condition, we can get the input signal expression is as shown in Equation(1). When the differential input voltage $U_{\mathrm{AB}} \geq 200 \mathrm{mV}$, it is high level; when $U_{\mathrm{AB}} \leq-200 \mathrm{mV}$, it is low level; otherwise, the bus in a random state.

$$
\left\{\begin{array}{l}
U_{\mathrm{AB}} \leq-200 \mathrm{mV} \quad \ldots \ldots \mid \text { ogi c } 0 \\
U_{\mathrm{AB}} \geq 200 \mathrm{mV} \quad \ldots \ldots \mid \text { ogi c } 1 \\
-200 \mathrm{mV} \leq U_{\mathrm{AB}} \leq 200 \mathrm{mV} \cdots \cdots \text {. unst abl e }
\end{array}\right.
$$

(2) The minimum input resistance

Under the standard conditions, the input impedance of RS485 interface chip is $12 \mathrm{k} \Omega$, and in this context, the maximum bus load is 32 . Therefore, the minimum common-mode input resistance for the bus is: $R_{\mathrm{CM}}=12 \mathrm{k} \Omega / 32=375 \Omega$. Therefore, the parallel resistance of $R_{\mathrm{B}}$ and $R_{\mathrm{In}}$ should be equal to the common-mode input resistance, and satisfy the Equation(2).

$$
R_{\mathrm{B}} \| R_{\mathrm{In}}=R_{\mathrm{CM}} \geq 375 \Omega
$$

Equation(2) also could be expressed as:

$$
\frac{1}{R_{\mathrm{B}}}+\frac{1}{R_{\mathrm{In}}}=\frac{1}{R_{\mathrm{CM}}} \leq \frac{1}{375 \Omega}
$$

\section{Solving the Key Parameters of RS485 Fieldbus}

\subsection{The Range of Bias Resistor}

After adding bias resistors $R_{\mathrm{B}}$, the parallel value of $R_{\mathrm{B}}$ and the equivalent input resistance $R_{\mathrm{In}}$, must satisfy the condition of inequality(2), which indicates that $R_{\mathrm{B}}$ should have a minimum value. On the other hand, the addition of $R_{\mathrm{B}}$ degrades the driver ability of RS485 fieldbus if the value of $R_{\mathrm{B}}$ is too large, which implies that $R_{\mathrm{B}}$ should have a maximum value. Next, we will solve the minimum value of $R_{\mathrm{B}}$ based on the KCL law, and then figure out the maximum value $R_{\mathrm{B}}$ through the current limiting method.

(1) The minimum value of $R_{\mathrm{B}}$

Here, we adopt Kirchhoff's current law (KCL) to establish current equations of node A and B, as shown in Fig.4, the current that flows into one node equals the current flows out it. The solid line presents the current flows into node A, and dotted line shows the current flows from node A, the same situation as to node B. Equation (4) shows the KCL current equations of node $\mathrm{A}$ and $\mathrm{B}$ :

$$
\left\{\begin{array}{l}
\mathrm{A}: \frac{U_{\mathrm{S}}-U_{\mathrm{A}}}{R_{\mathrm{B}}}=\frac{U_{\mathrm{A}}-U_{\mathrm{B}}}{R_{\mathrm{T} 2}}+\frac{U_{\mathrm{A}}-U_{\mathrm{B}}}{R_{\mathrm{T} 1}}+\frac{U_{\mathrm{A}}}{R_{\mathrm{In}}} \\
\mathrm{B}: \frac{U_{\mathrm{A}}-U_{\mathrm{B}}}{R_{\mathrm{T} 2}}+\frac{U_{\mathrm{A}}-U_{\mathrm{B}}}{R_{\mathrm{T} 1}}=\frac{U_{\mathrm{B}}}{R_{\mathrm{In}}}+\frac{U_{\mathrm{B}}}{R_{\mathrm{B}}}
\end{array}\right.
$$

Where, $U_{\mathrm{S}}$ is bus voltage, $\mathrm{V}, U_{\mathrm{A}}$ and $U_{\mathrm{B}}$ are voltage of point $\mathrm{A}$ and $\mathrm{B}$ respectively, $\mathrm{V}$, $R_{\mathrm{B}}$ is bias resistor, $\Omega, R_{\mathrm{In}}$ is the equivalent input resistance for the bus, $\Omega, R_{\mathrm{T} 1}$ and $R_{\mathrm{T} 2}$ are the terminal resistors, $\Omega$. 


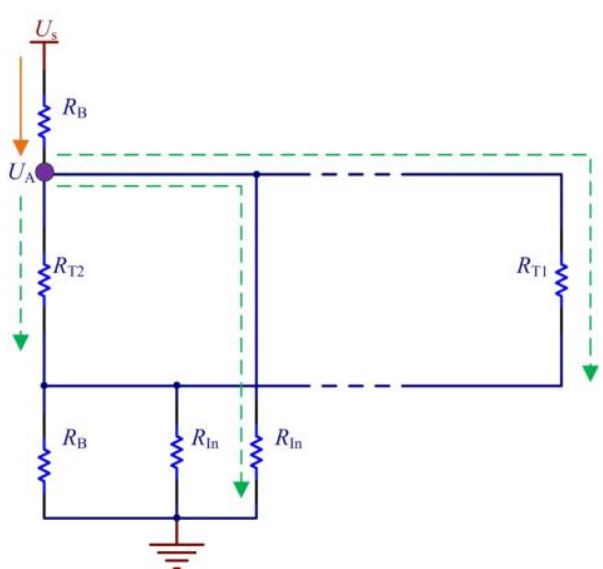

(a)

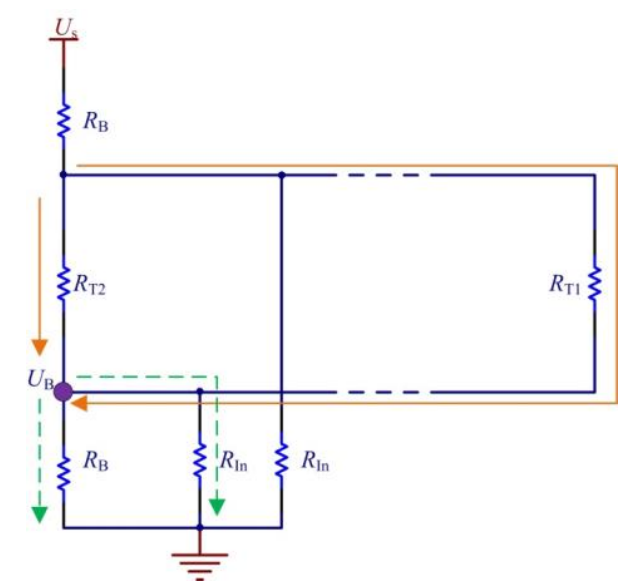

(b)

Figure 4. Current Flow in Failsafe Biasing Resistors Network

The respective line voltages $U_{\mathrm{A}}$ and $U_{\mathrm{B}}$ can be deduced from Equation(4), as follows:

$$
\begin{gathered}
U_{\mathrm{A}}=R_{\mathrm{In}}\left[\frac{U_{\mathrm{S}}-U_{\mathrm{A}}}{R_{\mathrm{B}}}-\left(U_{\mathrm{A}}-U_{\mathrm{B}}\right)\left(\frac{1}{R_{\mathrm{T} 1}}+\frac{1}{R_{\mathrm{T} 2}}\right)\right] \\
U_{\mathrm{B}}=R_{\mathrm{In}}\left[\left(U_{\mathrm{A}}-U_{\mathrm{B}}\right)\left(\frac{1}{R_{\mathrm{T} 1}}+\frac{1}{R_{\mathrm{T} 2}}\right)-\frac{U_{\mathrm{B}}}{R_{\mathrm{B}}}\right]
\end{gathered}
$$

The differential input voltage $U_{\mathrm{AB}}$ is then obtained, as shown in Equation(7).

$$
U_{\mathrm{AB}}=U_{\mathrm{A}}-U_{\mathrm{B}}=R_{\mathrm{In}}\left[\frac{U_{\mathrm{S}}-U_{\mathrm{AB}}}{R_{\mathrm{B}}}-2 U_{\mathrm{AB}}\left(\frac{1}{R_{\mathrm{T} 1}}+\frac{1}{R_{\mathrm{T} 2}}\right)\right]
$$

Simplify Equation(7) as following:

$$
U_{\mathrm{AB}}=\frac{U_{\mathrm{S}}}{R_{\mathrm{B}}} \frac{1}{2\left(\frac{1}{R_{\mathrm{T} 1}}+\frac{1}{R_{\mathrm{T} 2}}\right)+\frac{1}{R_{\mathrm{In}}}+\frac{1}{R_{\mathrm{B}}}}
$$

It is worth noting that the FSS must occur in the worst cases, namely, in the smallest power supply voltage and the maximum noise voltage conditions. Assume that, the supply voltage is $5 \mathrm{~V}$, with $\pm 5 \%$ tolerance, namely, the worst condition is $U_{\mathrm{S}}=4.75 \mathrm{v}$. We can assume that the input differential input voltage is as Equation(9).

$$
U_{\mathrm{AB}}=U_{\mathrm{IT}}+U_{\mathrm{N}}
$$

Where, the threshold voltage $U_{\text {IT }}$ is less than $200 \mathrm{mV}$, generally speaking, the worst noise voltage $U_{\mathrm{n}}$ is less than $50 \mathrm{mv}$, for a well-balance power system. There, we can get the corresponding value of $U_{\mathrm{AB}}$ is about $250 \mathrm{mV}$. In addition, before adding bias resistors $R_{\mathrm{B}}, R_{\mathrm{T} 1}$ and $R_{\mathrm{T} 2}$ equal to input bus impedance $Z_{0}(120) \Omega$. Now, the parallel resistance of $R_{\mathrm{T} 2}$ and the two bias resistors should be equal to the value of $R_{\mathrm{T} 1}$ in order to keep the symmetry of the circuit, which could be expressed as Equation(10).

$$
R_{\mathrm{T} 2} \| 2 R_{\mathrm{B}}=R_{\mathrm{T} 1}=\mathrm{Z}_{0}
$$

The Equation(10) also could be in the form as following:

$$
\frac{1}{R_{\mathrm{T} 2}}=\frac{1}{Z_{0}}-\frac{1}{2 R_{B}}
$$


Taken all these conditions into Equation(8), the minimum value of $R_{\mathrm{B}}$ could be solved, $R_{\mathrm{Bmin}}=556 \Omega$.

(2) Solving the maximum value of $R_{\mathrm{B}}$

Next, we will solve the maximum value of $R_{\mathrm{B}}$ by using current limiting method, and then obtain the range of $R_{\mathrm{B}}$. As we know, the differential input voltage must be satisfied $U_{\mathrm{AB}} \geq 200 \mathrm{mV}$ in order to enable the normal communication of RS485 fieldbus, which indicates that, there exits minimum drive current on the bus.

As shown in Fig.3, if there is no bias resistor, the parallel resistance of two terminal resistors equals to $\mathrm{Z}_{0} / 2$, namely $60 \Omega$. So, the overall difference load, $R_{\text {Diff }}$ can be expressed as:

$$
R_{\text {Diff }}=\frac{Z_{0}}{2} \square \frac{R_{\mathrm{Im}}}{N} \quad N=1,2, \cdots, 32
$$

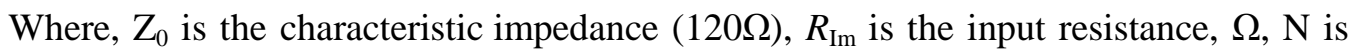
the number of nodes hang up on the bus. In this context, the minimum bias current is expressed as in Equation(13).

$$
I_{\text {min }}=\frac{U_{\mathrm{IT}}}{R_{\text {Diff }}} \quad N=1,2, \cdots, 32
$$

Based on this, the relevant maximum resistor of fieldbus is:

$$
R_{\text {max }}=\frac{U_{\mathrm{s}}}{I_{\text {min }}}=\frac{U_{\mathrm{s}}}{U_{\mathrm{IT}}}\left(\frac{Z_{0}}{2} \square \frac{R_{\mathrm{Im}}}{N}\right) \quad N=1,2, \cdots, 32
$$

Furthermore, the expression of maximum value of $R_{\mathrm{B}}$ is shown as in Equation(15).

$$
R_{\mathrm{B} \max }=\frac{R_{\text {max }}-R_{\text {Diff }}}{2}=\left(\frac{U_{\mathrm{s}}}{U_{\mathrm{IT}}}-1\right)\left(\frac{Z_{0}}{2} \square \frac{R_{\mathrm{Im}}}{N}\right) \quad N=1,2, \cdots, 32
$$

Where, $U_{\mathrm{S}}$ is bus voltage, $\mathrm{V}, U_{\mathrm{IT}}$ is the threshold voltage, $\mathrm{V}, \mathrm{Z}_{0}$ is the characteristic impedance, $\Omega, R_{\mathrm{Im}}$ is the input impedance, $\Omega, \mathrm{N}$ is the number of nodes hang up on the bus. It is not difficulty to see that, the $R_{\mathrm{Bmax}}$ is inversely proportional to the number of network nodes.

After adding $R_{\mathrm{B}}$, the communication stability of RS485 fieldbus is improved. However, this structure reduces the driver ability and makes the maximum number of nodes $N_{\max }$ decreased. There, the effect of $R_{\mathrm{B}}$ and the specific value of $N_{\max }$ should be further studied.

\subsection{Search for the Best Value of Bias Resistor}

The RS485 standard uses the concept of Unit Load (UL) to evaluate the driver ability, that is, the chip in 1UL must be able to drive 32 nodes, and the relevant input impedance is $12 \mathrm{k} \Omega$. By such analogy, the input impedance of $1 / 2 \mathrm{UL}$ is $24 \mathrm{k} \Omega$, the input impedance of $1 / 4 \mathrm{UL}$ is $48 \mathrm{k} \Omega$, and the input impedance of $1 / 8 \mathrm{UL}$ is $96 \mathrm{k} \Omega$. The relationship of $N_{\max }$ and $R_{\mathrm{B}}$ could be described as in Equation(16).

$$
F\left(N_{\max }\right)=f\left(R_{\mathrm{Im}}, R_{\mathrm{B}}\right)
$$

Where, $N_{\max }$ is the maximum value of network nodes, $R_{\mathrm{Im}}$ is the input impedance, $\Omega$, with its value $12 \mathrm{k} \Omega, 24 \mathrm{k} \Omega, 48 \mathrm{k} \Omega$ and $96 \mathrm{k} \Omega$. When the RS485 chip is determined, the relevant input impedance is determined too. $R_{\mathrm{B}}$ is the bias resistors, $\Omega$. Here, focus on the 1 UL driver chip as an example, the $N_{\max }$ should satisfy following Equation(17).

$$
\frac{N_{\max }}{32}=\frac{R_{\mathrm{CM}}}{R_{\mathrm{In}}}
$$


By transform the Equation(3), we could get the expression of $R_{\mathrm{In}}$, as shown in Equation(18).

$$
R_{\mathrm{In}}=\frac{1}{\frac{1}{R_{\mathrm{CM}}}-\frac{1}{R_{\mathrm{B}}}}
$$

Taken Equation(18) into Equation(17), and we could obtain the expression of $N_{\max }$, as shown in Equation(19).

$$
N_{\text {max }}=32 \frac{R_{\mathrm{CM}}}{R_{\mathrm{In}}}=32\left(1-\frac{R_{\mathrm{CM}}}{R_{\mathrm{B}}}\right) \quad 556 \Omega \leq R_{\mathrm{B}} \leq 716 \Omega
$$

Where, the value of $R_{\mathrm{B}}$ is in range $R_{\mathrm{Bmin}}$ and $R_{\mathrm{Bmax}}$. From Equation(19), $N_{\max }$ increases with the increasing of value of $R_{\mathrm{B}}$, which is an opposite conclusion to the Equation(15). In order to reflect the relationship of $R_{\mathrm{B}}$ and $N_{\max }$ more intuitive, the Equation(15) and Equation(19) are described through graphics format, as shown in Fig.5(a) and Fig.5(b), respectively.

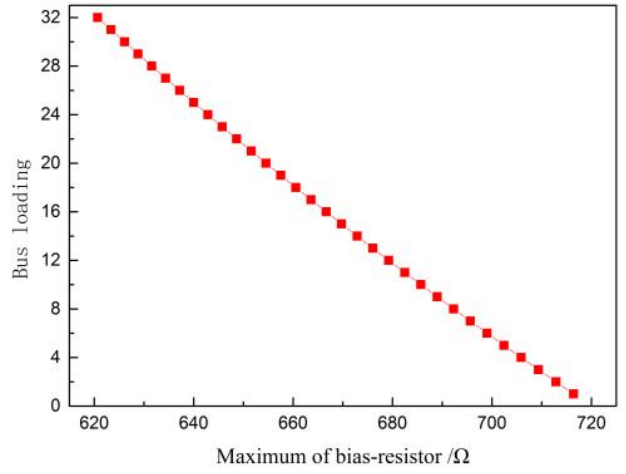

(a)



(b)

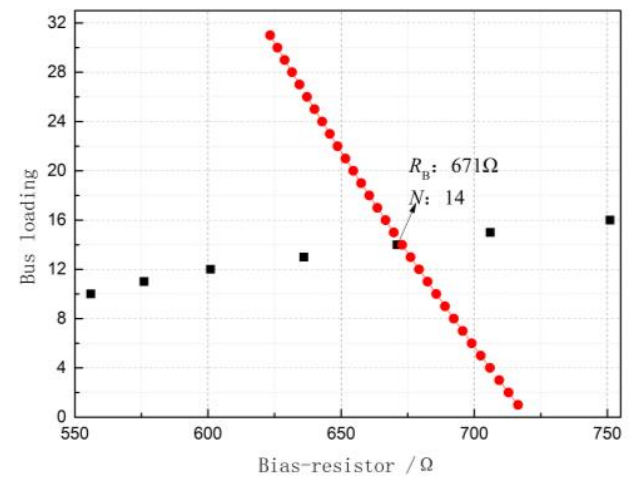

(c)

Figure 5. The Relationship between Bus Loading and Biasing Resistor

In Fig.5 (a), the upper limit of bias resistors declines from $716 \Omega$ to $620 \Omega$ linearly, with the network nodes gradually increasing. On the other hand, in Fig.5 (b), the value of maximum nodes increases along the increasing from $560 \Omega$ to $716 \Omega$, and the point in the top right corner $(751,16)$ is already insignificance. It is amazing that if we put the Fig.5 (a) and Fig.5 (b) together and get Fig.5 (c), the best value of bias resistor is found out at the intersection point position, $(671,14)$. By the same token, we are able to get the best point of $R_{\mathrm{B}}$ for $1 / 2 \mathrm{UL}, 1 / 4 \mathrm{UL}$ and $1 / 8 \mathrm{UL}$ scenes. The combination of best bias resistor and maximum nodes locations are $(671,28),(668,56)$ and $(672113)$, respectively. 


\section{Conclusions}

Adding bias resistors to the RS485 fieldbus is necessary to effectively eliminate the phenomenon of FSS and efficiently maintain the communication performance of RS485 in applications. To calculate the best value of bias resistor of RS485 fieldbus, this paper adopts KCS law and current limiting method, and solves the minimum value of $R_{\mathrm{B}}$ is $556 \Omega$, the maximum value of $R_{\mathrm{B}}$ is $716 \Omega$ (there is only one node on the bus). Furthermore, we get the best value of bias resistor through the relationship curves between $N_{\max }$ and $R_{\mathrm{B}}$. At the exact point, it can play the role of the bias resistor, safeguarding the RS485 fieldbus communication stability, and can get the maximum bus nodes number at the same time. The results of this paper can be used in designing of RS485 fieldbus application directly.

\section{Acknowledgments}

The present research is supported by the Dalian University of Technology Scientific research start-up funding (DUT14RC(3)123, DUT14RC(3)129).

\section{References}

[1] J. Zhang, Z.J. Wang, S.R. Zhu and X.M. Meng, "Design on distributed fault monitoring system for navigation lights", vol.263, no.12, (2013), pp.569-573.

[2] A. Jorge, J. Guerreiro, P. Pereira, J. Martins, and L. Gomes, "Energy Consumption Monitoring System for Large Complexes", Conference on Computing, Electrical and Industrial Systems, (2010).

[3] D.A. Antonov, K. K. Veremeenko, M. V. Zharkov and R. Zimin, "Experimental automobile integrated navigation module", vol.23, no.12, (2008), pp. 20-23.

[4] L. Zhao, J.L. Zhang and R.B. Liang, "Development of an energy monitoring system for large public buildings", vol.66, (2013), pp.41-48.

[5] Y. L. Jin and J. Q. Yang, "Design an intelligent environment control system for GreenHouse based on RS485 bus", The 2nd International Conference on Digital Manufacturing and Automation, (2011).

[6] Y. M. Li, "Technology study of new industry data collection and monitoring system", Proceedings of 2011 Conference on Electronic and Mechanical Engineering and Information Technology, (2011).

[7] J. Zhang, Y. Mei, S.H. Miao and P. Liu, "Measures to enhance the reliability of RS485 fieldbus network", vol.23, no.1, (2004), pp.31-33.

[8] F.E. Zhang, J.S. Heng and H,Q. Zhen, "Research on reliability of RS485 bus network", vol.27, no.6, (2006), pp.2458-2459.

[9] Y.S. Yu and W.Q. Ye, “Application research on reliability of RS485 bus” RS-485, vol.23, no.8, (2007), pp.274-276.

[10] X.B. Zhao and X.F. Zhou, "Anti-interference design of RS485 bus in coal mine safety”, vol.39, no.2, (2013), pp. 83-86.

[11] L. Zhao, J.L. Zhang and R.B. Liang, "The Solving of Bias Resistor and Its Effect on the RS485 Fieldbus", vol.2, no.1, (2014), pp.71-75.

[12] Y.L. Yu, "Analys is of signal integrity in the design of digital circuits", vol.27, no.7, (2004), pp.843846. 